“ (C) 2017 IEEE. Personal use of this material is permitted. Permission from IEEE must be obtained for all other uses, in any current or future media, including

reprinting/republishing this material for advertising or promotional purposes, creating new collective works, for resale or redistribution to servers or lists, or reuse of any copyrighted component of this work in other works." 


\title{
A Wideband Multilayer Substrate Integrated Waveguide (SIW) Cavity-Backed Slot Antenna Array
}

\author{
Yang Cai, Yingsong Zhang, Can Ding, and Zuping Qian
}

\begin{abstract}
In this paper, a wideband $2 \times 2$ multilayer substrate integrated waveguide (SIW)-based cavity-backed slot array is proposed. The array element is constructed by stacking five layers of SIW cavity-backed slots and has a wide impedance bandwidth from 18 to $30 \mathrm{GHz}$. Two different feed networks based on probe coupling and slot coupling are introduced to maintain the wideband characteristic of the proposed element when employed in an array. Two $2 \times 2$ arrays fed by the proposed feed networks are designed, fabricated, and tested. The measured results show that both the arrays employing probe coupling and slot coupling feed networks have a wide impedance bandwidth over $30 \%$. Within the obtained operation bands, good radiation performance is achieved. Moreover, advantages and disadvantages of the proposed two feed networks are discussed.
\end{abstract}

Index Terms-Antenna array, coupling, cavity-backed slot antenna, substrate integrated waveguide (SIW), wideband.

\section{INTRODUCTION}

$\mathbf{S}$ UBSTRATE intergrated waveguide (SIW), which is firstly proposed in [1] as a form of transmission lines, has advantages of low cost, low loss, simple fabrication process and easy integration with planar circuits. Since then, SIW has been extensively used in antenna designs. Cavity-backed slot antennas based on SIW have attracted many attentions and have been proved to be useful in many applications due to its compact size, low cost, and low loss [2]. In particular, this type of antennas is very suitable to be used in constructing arrays to achieve high gains for the following reasons. Firstly, SIW cavities functioning as slot radiators can also be used as feed lines at the same time, which can eliminate the need of additional feed network and results in compact size and low loss [3]. Secondly, the closed-form SIW structure provides better isolation between array elements [4]. This significantly reduces the complexity of array synthesis. Last but not least, array feed networks based on SIW usually have lower losses than those built using microstrips [5]. However, compared to

This work was supported in part by the National Science Foundation of China under Grant 61271103, and in part by the Open Project of State Key Laboratory of Millimeter Waves, Southeast University China under Grant K201505.

Y. Cai, Y.-S. Zhang, and Z.-P. Qian are with the College of Communications Engineering, PLA University of Science and Technology, Nanjing 210007, China (email: caiyang_1991@163.com).

Y. S. Zhang is also with the State Key Laboratory of Millimeter Waves, Southeast University, Nanjing 210096, China (e-mail: yszhangche@ 126.com).

C. Ding is with Global Big Data Technologies Centre (GBDTC), University of Technology, Sydney (UTS), Sydney, NSW, 2007, Australia (email: can.ding.1989@gmail.com)

Manuscript received ; revised other forms of antennas like bow-tie antennas, slot antennas, the narrower bandwidth is the limitation [6]-[11].

As well known, the bandwidth of an array is highly dependent on the bandwidth of the employed element. However, SIW cavity-backed slot antennas typically have intrinsically narrow impedance bandwidths. Several representative methods were proposed in the past few years to enhance the bandwidth. In [12], a via-hole was placed above the slot to create an additional resonance, which broadened the bandwidth to $3.7 \%$ in comparison with that without loading. In [13], the bandwidth of a SIW cavity-backed slot antenna was enhanced to $6.3 \%$ by exciting hybrid SIW cavity modes. Through increasing the width to length ratio of the wide slot, dual resonances of SIW cavity-backed slot antenna were generated and $11.6 \%$ bandwidth was achieved [3], which is the widest impedance bandwidth achieved by a single-layered SIW cavity-backed slot antenna so far. Besides, when the cavity-backed slot antenna was loaded with an open-ended cavity, the impedance bandwidth of a single element was enhanced to $23 \%$ [14].

Significant progresses have been made on the bandwidth enhancement for the SIW cavity-backed slot antennas. However, we couldn't expect an array can have a similar bandwidth as the employed wideband array element without a welldesigned feed network. To obtain a wideband SIW-based slot array, the feed network plays the role as important as the array elements do. Shunt-feed approach proposed in [3][4] is a popular method to feed cavity-backed slot arrays and can offer excellent radiation performance. However, this approach exhibits limited bandwidth since the feed network and radiators share the same substrate layer. In [15], a $4 \times 4$ SIW cavity-backed slot array with a wide bandwidth of $14 \%$ was proposed using the SIW cavities themselves as the feed network. Then the array was expanded to a $16 \times 16$ array with a specifically designed corporate feed network, maintaining a similar bandwidth of $15 \%$ [16]. In [17], an SIW higher order mode cavity-backed slot antenna array with simplified feed network was presented. The slots were arranged asymmetrically in the cavity to introduce new resonances and $16.7 \%$ bandwidth was obtained.

In this paper, a wideband four-element SIW-based slot antenna array is proposed. The array element is constructed by stacking five-layers of SIW cavity-backed slots and has a wide operation band. Two methods are then introduced to feed a $2 \times 2$ array based on the proposed multilayer slot antenna. The resultant two arrays are also fabricated and tested. The tested results show that both of the obtained arrays have the 


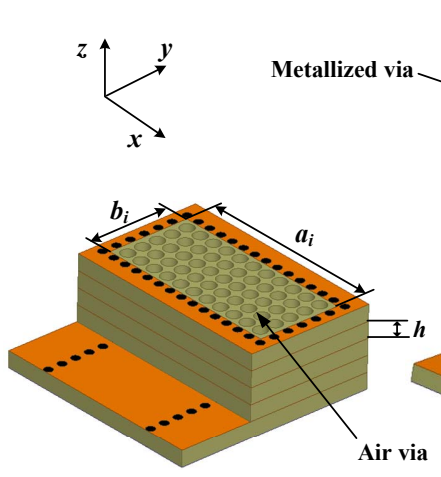

(a)

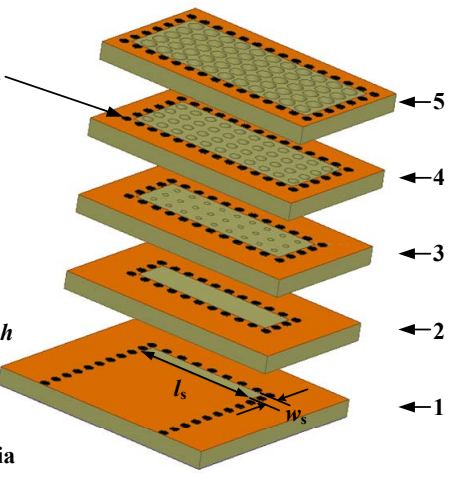

(b)
Fig. 1. Geometry of the array element. (a) Whole view. (b) Anatomy view.

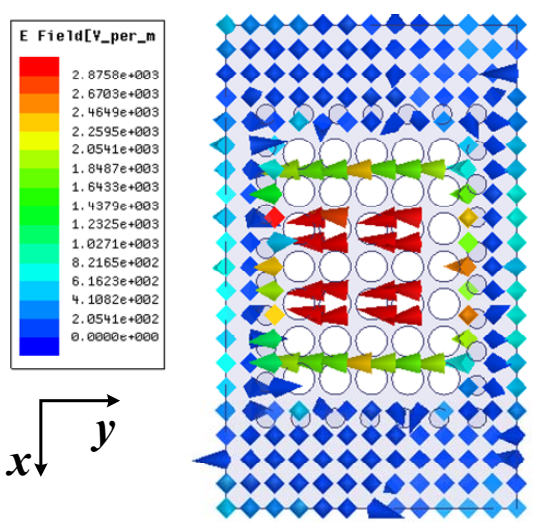

Fig. 2. The electric field distribution on the element aperture at $25 \mathrm{GHz}$.

bandwidth of over $30 \%$. Moreover, the two feed networks are compared to demonstrate their strength and weakness.

\section{ELEMENT Design}

Fig. 1 depicts the configuration of the proposed SIW cavitybacked slot antenna as the array element. The antenna consists of five layers, with each layer is based on Rogers RT/Duroid 5880 PCB board with permittivity of 2.2 , thickness of 1.524 $\mathrm{mm}$, and loss tangent of 0.0009 . On the bottom layer, a transverse slot with a size of $w_{s} \times l_{s}$ is etched at the end of a SIW feed line, which is the driven element. Four gradually expanded SIW cavities are stacked and placed above the driven slot to direct the radiation. Metallized posts with diameter of $d$ are inserted into the substrates to construct SIW cavities. The size of the cavity in the $i$ th layer is $a_{i} \times b_{i}(i=2,3,4,5)$. Air vias are drilled on layers 3,4 , and 5 to manipulate the substrate permittivity by adjusting the diameters $\left(D_{3}, D_{4}, D_{5}\right)$ of the vias. With the optimized air vias, the proposed structure has a gradually reduced permittivity from the bottom layer to the top layer. This mitigates the permittivity change between the radiator and the air, thereby minimizing the reflection when the wave propagates into the air from the substrate. The employed air vias play a significant role in matching the antenna and widening the bandwidth. Detailed design principal of the air vias can be found in our previours work [18][19].

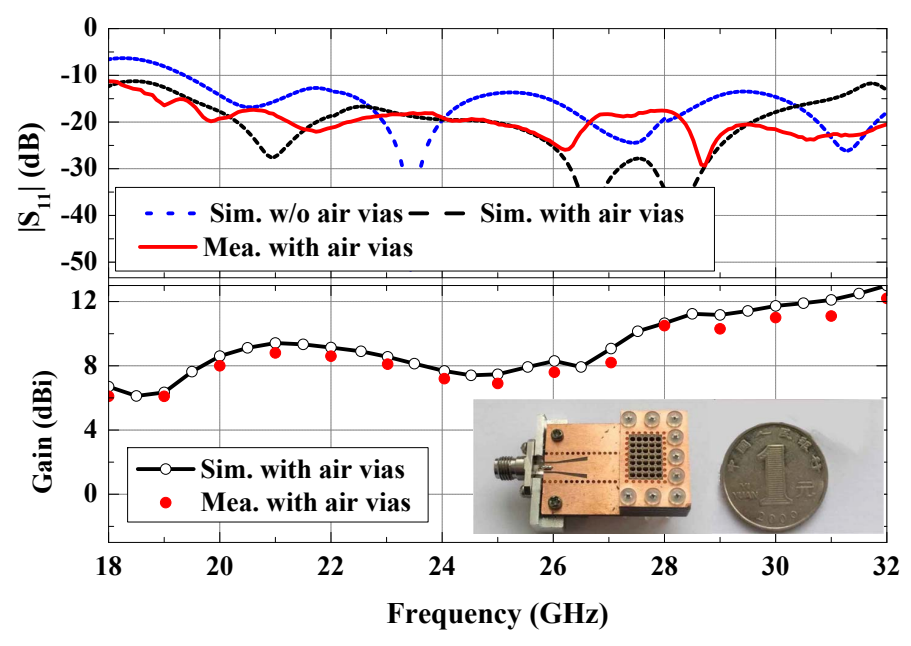

Fig. 3. Simulated and measured reflection coefficient $\left|S_{11}\right|$ and realized gain of the proposed antenna.

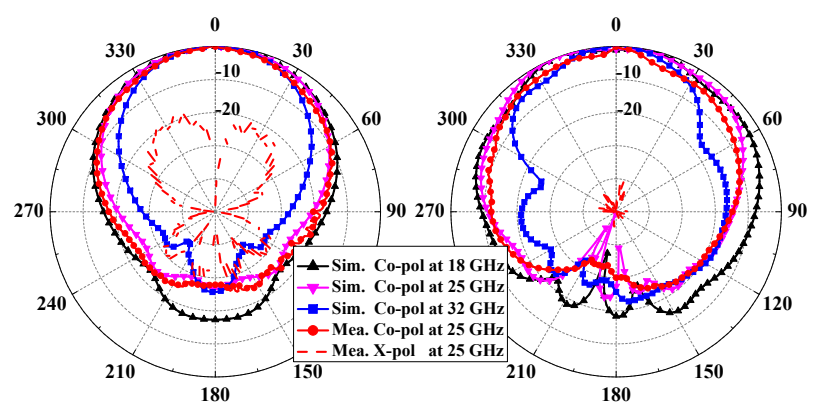

(a)

(b)

Fig. 4. Far field radiation patterns of the antenna element at three representing frequencies. (a) H-plane. (b) E-plane.

TABLE I

PARAMETERS OF THE ARRAY ElEMENT (UNIT: MM).

\begin{tabular}{cc|cc|cc|cc}
\hline \hline$a_{1}$ & 8.6 & $b_{1}$ & 3.1 & $a_{2}$ & 10.2 & $b_{2}$ & 4.7 \\
\hline \hline$a_{3}$ & 11.8 & $b_{3}$ & 6.3 & $a_{4}$ & 11.8 & $b_{4}$ & 7.9 \\
\hline \hline$l_{s}$ & 7 & $w_{s}$ & 1.5 & $h$ & 1.524 & $d$ & 1.6 \\
\hline \hline$D_{3}$ & 0.3 & $D_{4}$ & 0.75 & $D_{5}$ & 1.2 & & \\
\hline \hline
\end{tabular}

The dimensions of the slot radiator, cavities, and air vias are optimized to get the widest impedance bandwidth and a directional radiation pattern. The optimized dimension values are listed in Table I. A prototype was fabricated and measured, as shown in Fig. 3. A 50-Ohms-coplanar waveguide (CPW) is adopted to facilitate the measurement of antenna performance. The electric field distribution on the element aperture at 25 $\mathrm{GHz}$ is shown in Fig. 2. It is observed that the proposed antenna is linearly polarized along y-axis. Fig. 3 plots the reflection coefficients $\left(\left|S_{11}\right|\right)$ and realized gains of the proposed slot antenna with and without the air vias. As observed in the figure, the slot antenna has a wide impedance bandwidth with the stacked SIW cavities and the matching is then significantly improved by loading the air vias. A broadside gain varying from $6.1 \mathrm{dBi}$ to $12.2 \mathrm{dBi}$ is obtained in the band ranging from 


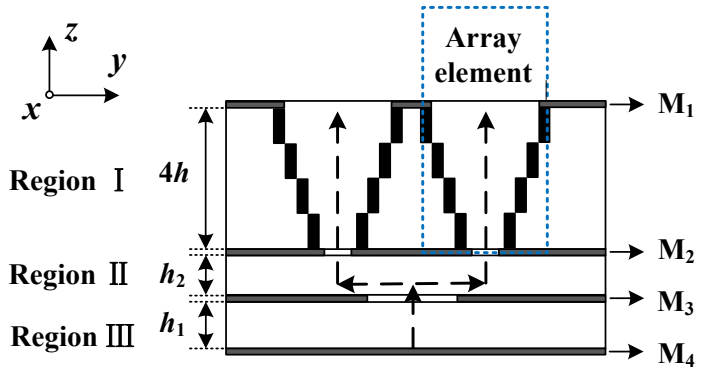

(a)

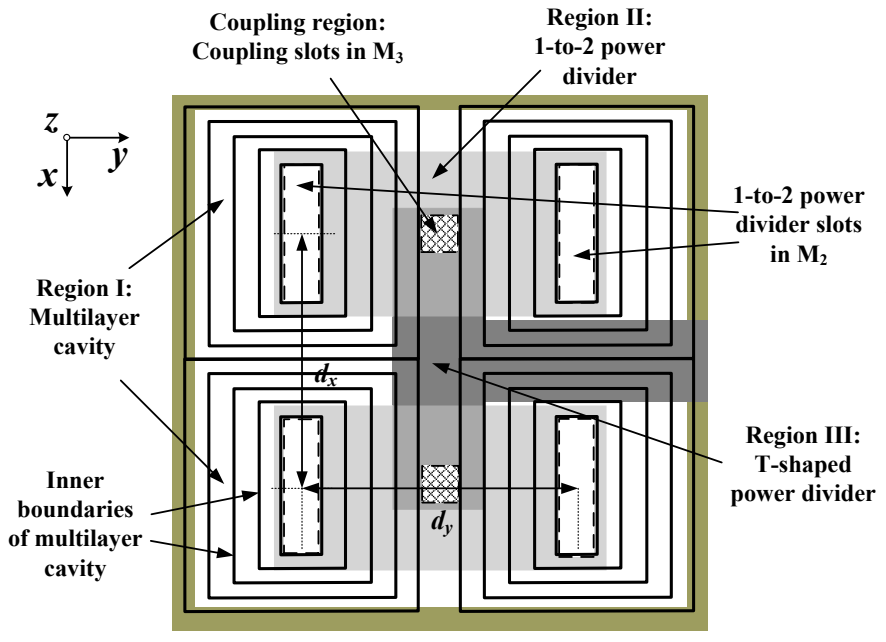

(b)

Fig. 5. Geometry of the antenna array. (a) Side view. (b) Top view.

18 to $32 \mathrm{GHz}$. Far-field radiation patterns at 18, 25, and 32 $\mathrm{GHz}$ in the $\mathrm{H}$ - and E-planes (xz- and yz-planes) are plotted in Figs. 4 (a) and 4 (b), respectively. Due to unavoidable leakage from the feeding CPW, radiation patterns in E-plane are affected in some degree. Nevertheless, stable broadside radiation pattern is obtained within the band ranging from 18 to $32 \mathrm{GHz}$. Moreover, the on-axis cross polarizations are lower than $20 \mathrm{~dB}$ and this antenna maintains reasonably good polarization discrimination at the main radiation direction as well. Above all, in comparison with the conventional SIW cavity backed slot antennas [2][3][13], the operating bandwidth is significantly broadened.

\section{Design of the Multilayer Cavity-Backed Slot ANTENNA ARRAY}

Based on the proposed wideband slot antenna, we aim to construct a slot antenna array having similar bandwidth. The challenge comes from the design of its feed network that should have a wideband characteristic and compact size.

A $2 \times 2$ array based on the proposed SIW slot antenna and its feed network are presented in Fig. 5 in a schematic way. Figs. 5 (a) and 5 (b) depict the side view and top view of the antenna array, respectively. The array is constructed by stacking six PCB layers. From top to bottom, the first four layers, defined as Region I, are the gradually expanded SIW cavities placed atop the radiating slot to widen the bandwidth. The cavity-backed slot radiators are located on

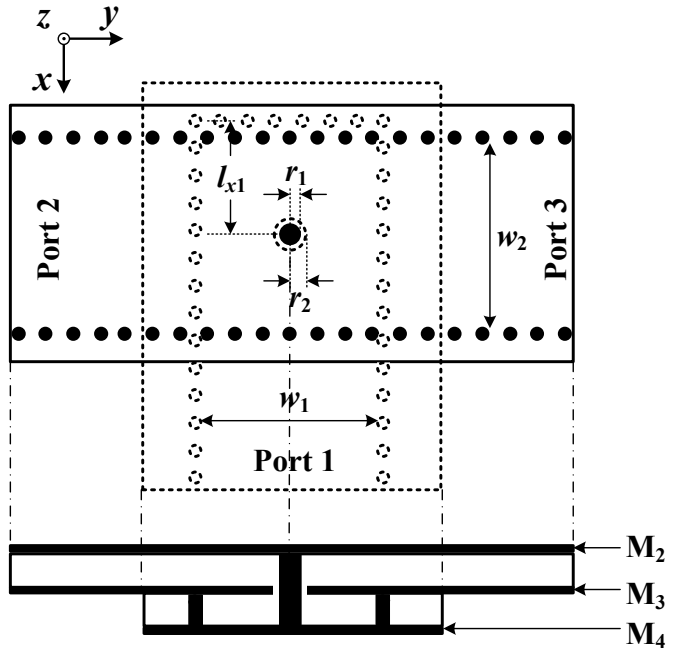

Fig. 6. Configuration of the 1-to-2 power divider based on probe coupling.

TABLE II

PARAMETERS OF THE POWER DIVIDER. (UNIT: MM).

\begin{tabular}{llllllll}
\hline$w_{1}$ & 7 & $w_{2}$ & 7 & $r_{1}$ & 0.5 & $r_{2}$ & 0.9 \\
\hline$l_{x 1}$ & 3 & $h_{1}$ & 0.508 & $h_{2}$ & 1.524 & & \\
\hline
\end{tabular}

the fifth layer (Region II). On the sixth layer (Region III), there is a T-shaped 1-to-2 power divider. The energy can be equally divided to the four slots by properly adjusting the coupling between Regions II and III. The signal propagating trace in this antenna is represented by the dashed lines with arrows. The layers $M_{1}$ to $M_{4}$ denote the metal layers between substrate layers. As illustrated in Fig. 5 (b), Region I is constituted by the four multilayer SIW cavities. The distances between adjacent cavities in $\mathrm{x}$ - and $\mathrm{y}$-directions are $d_{x}$ and $d_{y}$, respectively. The multilayer cavities in Region I are directly excited by the four slots of the two 1-to-2 power dividers in Region II. The output branches of the T-shaped power divider in Region III are placed under the SIW cavities hosting the slots, thereby constituting second-stage power dividers through proper coupling, as indicated by two coupling regions represented by dashed squares. In order to clearly show the configuration of the multilayer structure, metal layers are not full depicted in Fig. 5 (b). As introduced later, the coupling regions can be realized through coupling probes or slots. In this work, two methods based on probe coupling and slot coupling are proposed to realize the feed network. The work mechanisms and design details are presented in the following subsections.

\section{A. Array Using Probe Coupling}

1) Design of the Probe Coupler: Fig. 6 shows perspective and side views of feed network based on probe coupling. As shown in the figure, the two SIW lines in Regions II and III are placed perpendicularly and coupled through a probe with radius of $r_{1}$. The probe is located in the centre of the SIW line in Region II, so that the power is equally divided into the two output ports. Meanwhile, the probe also lies on the vertical 


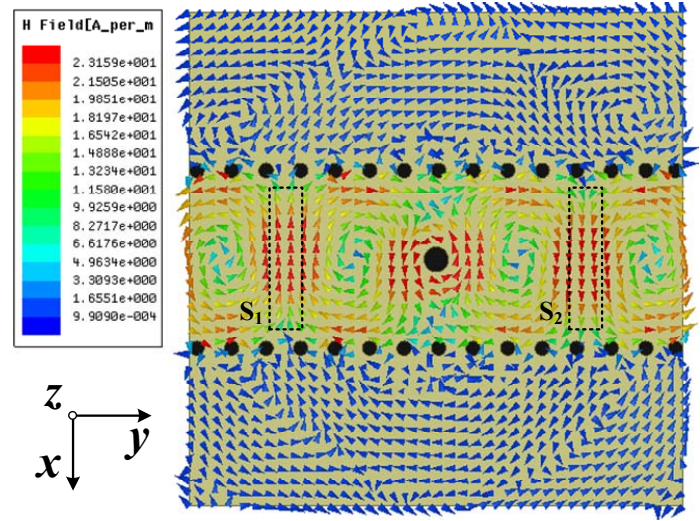

Fig. 7. Magnetic field distribution on top SIW of the probe coupler at 24 GHz.

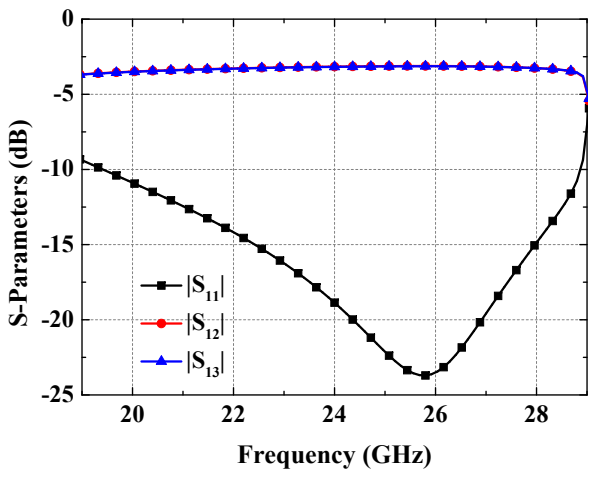

(a)

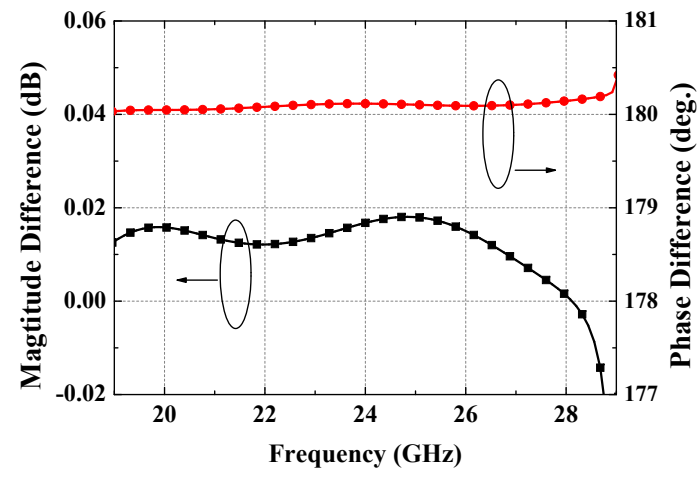

(b)

Fig. 8. Simulated results of the 1-to-2 power divider based on probe coupling. (a) S-parameters. (b) Magnitude and phase imbalances.

symmetry axis and has a distance of $l_{x 1}$ between the probe and the end of the feeding SIW in Region III. The distance $l_{x 1}$ should be set as $(2 n+1) \lambda_{g} / 4$, where $n=0,1,2, \ldots$, and $\lambda_{g}$ is the guided wavelength at the designed centre frequency. A circular slot with radius of $r_{2}\left(r_{2}>r_{1}\right)$ is etched on metal layer $M_{3}$ to avoid unwanted shorting. Good transmission characteristics of the power divider are obtained by tuning $w_{1}$, $w_{2}, r_{1}, r_{2}$, and $l_{x 1}$. The optimized values of these dimensions are listed in Table II.

The magnetic field distribution on the SIW cavities hosting slot radiators in Region II is plotted in Fig. 7. It is observed that the magnetic field of the $T E_{10}$ mode is symmetric respect

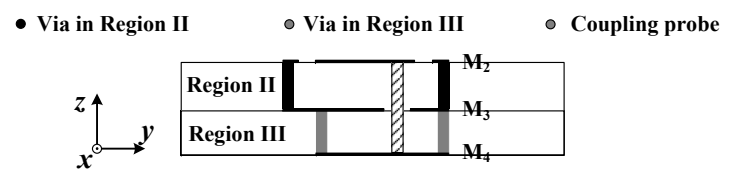

(a)

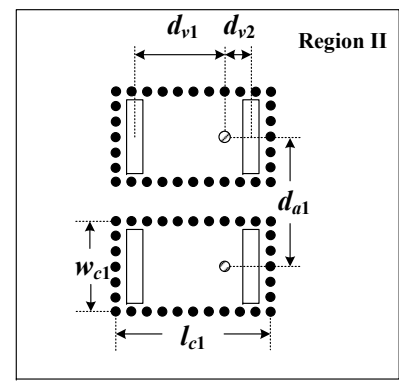

(b)

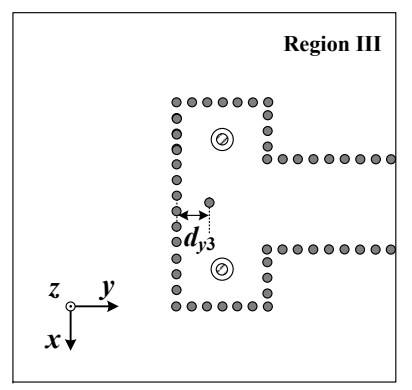

(c)
Fig. 9. Geometrical configuration of the feed network. (a) Side view. (b) Top view of Region II. (c) Top view of Region III.

to the center of the inserted probe. This leads to reversed magnetic field distributions at the two slots (namely the slots $S_{1}$ and $S_{2}$ labelled in Fig. 7). The reversed magnetic field distributions indicate that the receiving signals at Port 2 and Port 3 are out-of-phase. Simulated S-parameters of the power divider shown in Fig. 6 are plotted in Fig. 8. As shown in Fig. 8 (a), the power divider has a $\left|S_{11}\right|$ below $-10 \mathrm{~dB}$ in the frequency range from 19.5 to $28.9 \mathrm{GHz}$. Fig. 8 (b) shows that the output signals of the power divider are equal in magnitude but out-of-phase across the obtained band.

2) Design of Four Elements Array: Due to the fact that output signals of the power divider based on the probe coupler are out-of-phase, the slot radiators should be arranged asymmetrically respect to the coupling probe. That is to say, the slot radiators have to be translated away from the positions shown in Fig. 7. The resultant feed network of the antenna array consisting both Regions II and III is illustrated in Fig. 9. As shown in Fig. 9 (a), every two rectangular slots form a subarray and are surrounded by a cavity with size of $w_{c 1} \times l_{c 1}$. The adjacent slots are horizontally and vertically spaced by $d_{v 1}+d_{v 2}$ and $d_{a 1}$, respectively, where $d_{v 1}+d_{v 2} \approx \lambda_{0}, d_{a 1} \approx \lambda_{0}$. By adjusting the values of $d_{v 1}$ and $d_{v 2}$, the currents on the two slots in the same cavity can be identical in both magnitude and phase. As illustrated in Fig. 9 (b), a conventional T-shaped 1-to-2 power divider is designed in Region III to feed the two second-stage 1-to-2 power dividers. A metal post of diameter $1.6 \mathrm{~mm}$ is inserted in the cavity with a distance of $d_{y 3}$ to the sidewall to realize good transmission characteristic of the T-shaped power divider.

3) Experimental Results: Based on the above analyses, a prototype of the obtained array with the dimensions listed in Table III is fabricated and measured. Fig. 10 shows the photograph of the fabricated prototype. As shown in Fig. 10, several aligning holes are perforated in each layer to facilitate the assemblage of the multilayer array. The array is connected to a SMA through a transition from the SIW input in Region III to a 50-Ohms-microstrip line to facilitate the measurement of antenna performance. 
TABLE III

PARAMETERS OF THE ANTENNA ARRAY BASED ON PROBE COUPLING. (UNIT: MM)

\begin{tabular}{cc|cc|cc|cc}
\hline \hline$a_{1}$ & 7.7 & $b_{1}$ & 3.8 & $a_{2}$ & 9.3 & $b_{2}$ & 5.4 \\
\hline \hline$a_{3}$ & 10.9 & $b_{3}$ & 7 & $a_{4}$ & 10.9 & $b_{4}$ & 8.6 \\
\hline \hline$d_{v 1}$ & 7.7 & $d_{v 2}$ & 2.3 & $w_{c 1}$ & 7.8 & $l_{c 1}$ & 13.3 \\
\hline \hline$h$ & 1.524 & $d_{a 1}$ & 10.7 & $w_{s}$ & 1.5 & $l_{s}$ & 6.4 \\
\hline \hline$d_{y 3}$ & 2.4 & & & & & & \\
\hline \hline
\end{tabular}

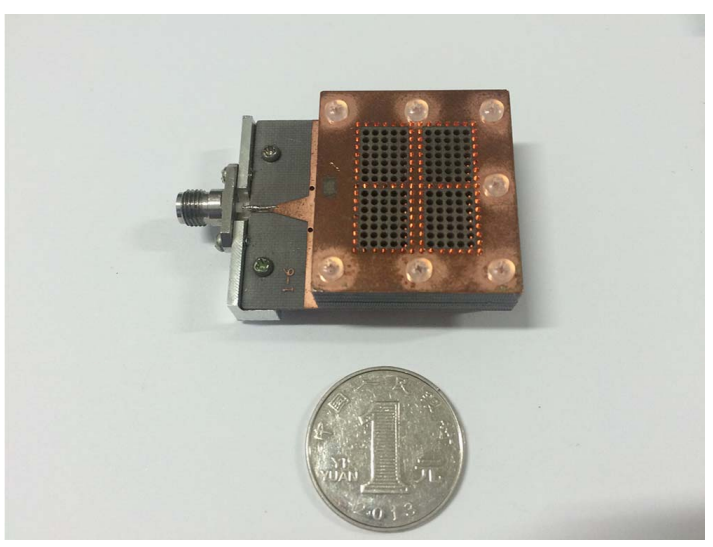

Fig. 10. Photograph of the fabricated array using probe coupling.

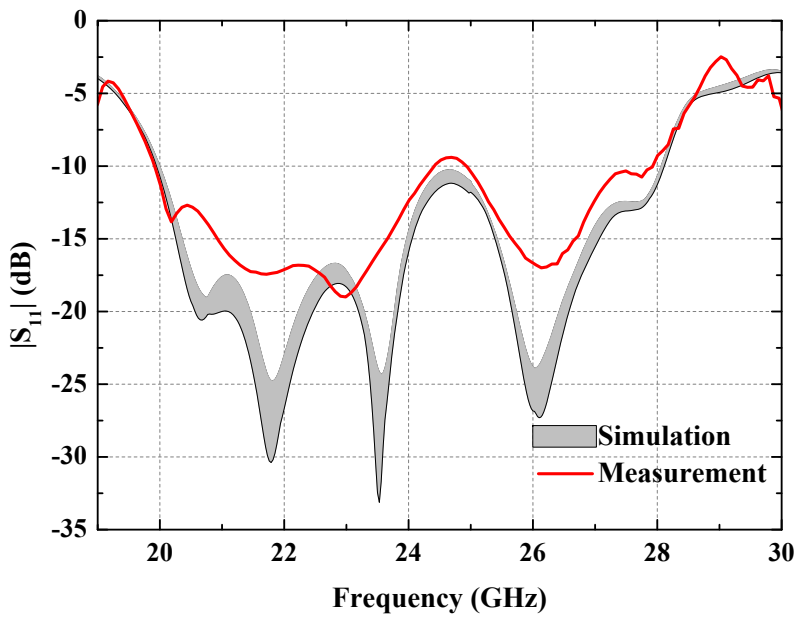

Fig. 11. Simulated and measured $\left|S_{11}\right|$ s of the four elements array using probe coupling.

The simulated and measured reflection coefficients of the resultant array are compared in Fig. 11. The grey area shows the simulated $\left|S_{11}\right|$ including the miss alignment between the probe coupler and the remaining substrates with a range from -0.1 to $0.1 \mathrm{~mm}$, which is the fabrication tolerance for the fabricated array. When fabrication tolerance is taken into consideration, the reflection coefficient is degraded in some degree but still below $-10 \mathrm{~dB}$ over the operating band, which demonstrates the robustness of the proposed array to the fabrication error. It is observed that the simulated and measured results are generally in good agreement, achieving a

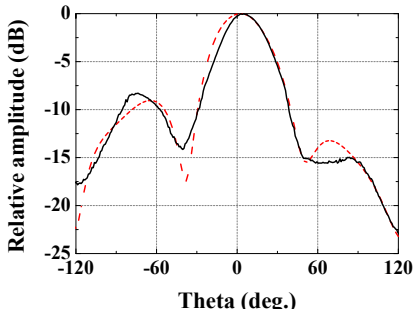

(a)

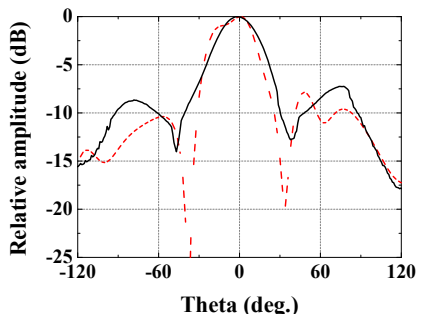

(c)

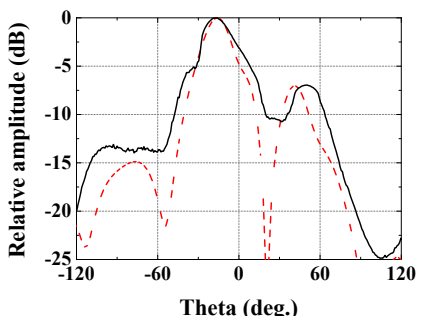

(e)

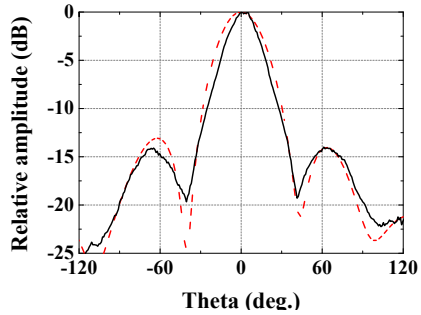

(b)

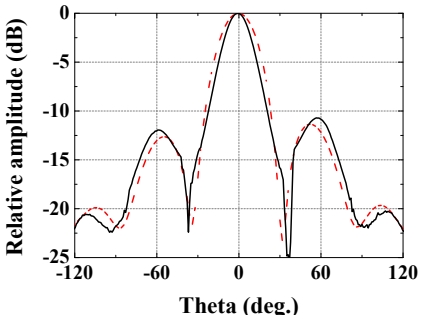

(d)

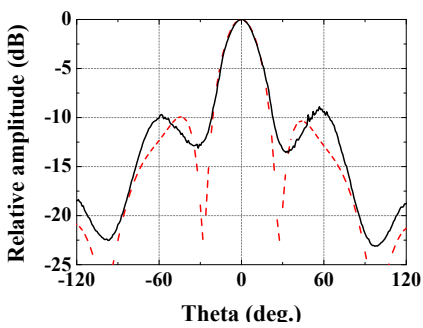

(f)
Fig. 12. Simulated and measured radiation patterns of the fabricated antenna at three different frequencies. (a) $20 \mathrm{GHz}$ in E-plane. (b) $20 \mathrm{GHz}$ in H-plane. (c) $24 \mathrm{GHz}$ in E-plane. (d) $24 \mathrm{GHz}$ in H-plane. (e) $28 \mathrm{GHz}$ in E-plane. (f) 28 $\mathrm{GHz}$ in H-plane. (Dashed lines denote the simulated results while solid lines denote measured ones.)

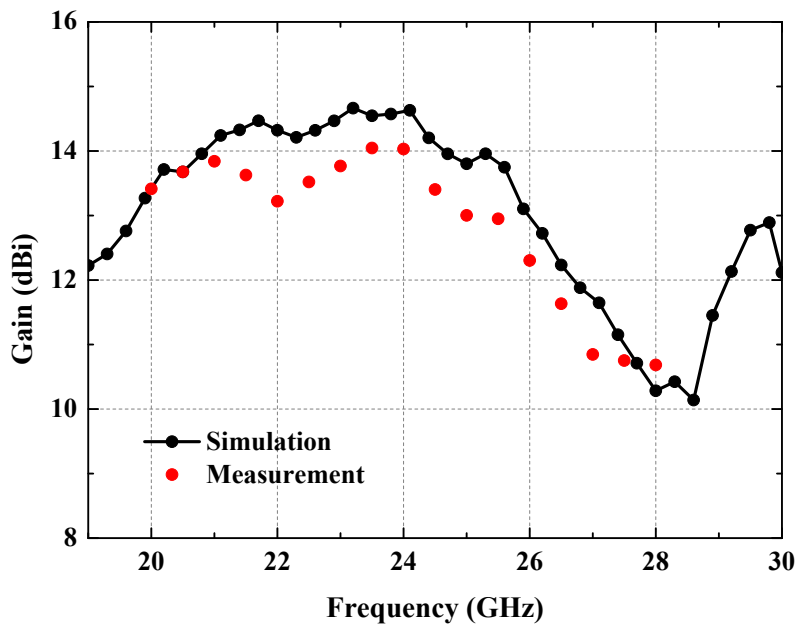

Fig. 13. Simulated and measured gains of the four elements array in broadside direction.

wide impedance bandwidth over $30 \%$ ranging from 20 to 28 $\mathrm{GHz}$. The discrepancy between the simulated and measured results mainly attributes to the air gaps between substrates.

Fig. 12 depicts the simulated and measured radiation patterns in $\mathrm{E}$ - and $\mathrm{H}$-planes at three representing frequencies. 


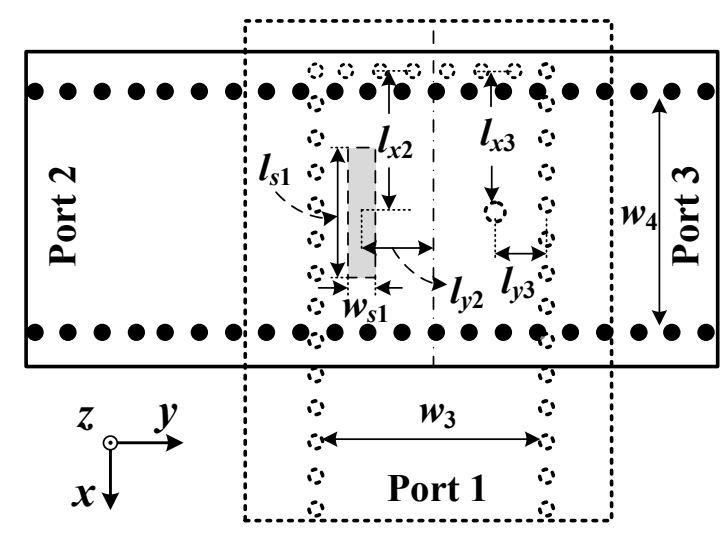

Fig. 14. Top view of the 1-to-2 power divider based on slot coupling.

As shown in the figures, the measured results show good accordance with the simulated ones. We remark that the main beam is shifted away from the broadside direction in the E-plane when the antenna working at high frequencies around $28 \mathrm{GHz}$. This is due to the fact that the elements are unsymmetrical with regards to the coupling probe in ydirection. As shown in Fig. 12, when the operating frequency goes high to $28 \mathrm{GHz}$, the mainlobe direction in E-plane is shifted over 15 degrees from broadside. Such phenomenon will decrease the broadside gain especially at high frequency in the designed band. Meanwhile, the main lobe direction in $\mathrm{H}$-plane still keeps pointing to the broadside direction with the varying frequency. The simulated and measured gains are plotted in Fig. 13. Due to the mismatch caused by air gaps between substrates, the measured gain is slightly lower than the simulated result. Besides, resulted by the shifted mainlobe, the broadside gains drop significantly at frequency higher than $26 \mathrm{GHz}$.

As described above, an effective method to build an SIW antenna array with wideband characteristic is proposed. Nevertheless, the radiating performance of the wideband antenna array could be further enhanced by employing symmetrical feeding method.

\section{B. Antenna Array Using Slot Coupling}

1) Design of the Slot Coupler: Fig. 14 shows part of feed network based on slot coupling. This power divider also consists of two layers but exhibits much difference in coupling mechanism in comparison with the previously introduced one. As shown in the figure, the two SIW lines in Regions II and III are also placed perpendicularly but coupled through a slot with size of $l_{s 1} \times w_{s 1}$. The slot is located in the centre of the SIW line in Region II to divide the power equally into the two output ports. Besides, the slot is set with an offset distance $l_{y 2}$ from the symmetrical line and has a distance of $l_{x 2}$ between the slot and the end of the feeding SIW in Region III. The distance $l_{x 2}$ should also be set as $(2 n+1) \lambda_{g} / 4$. An additional via is placed with distances $l_{y 3}$ and $l_{x 3}$ from sidewall and endwall to further improve the impedance matching of the power divider. The optimized dimension values are listed in Table IV.
TABLE IV

PARAMETERS OF THE POWER DIVIDER BASED ON SLOT COUPLING. (UNIT: MM).

\begin{tabular}{llllllllll}
\hline$w_{s 1}$ & 1.0 & $l_{s 1}$ & 5.2 & $l_{x 2}$ & 2.8 & $l_{y 2}$ & 1.5 & $h_{1}$ & 1.016 \\
\hline$w_{3}$ & 6.2 & $w_{4}$ & 6.2 & $l_{x 3}$ & 2.8 & $l_{y 3}$ & 1.5 & $h_{2}$ & 1.524 \\
\hline
\end{tabular}

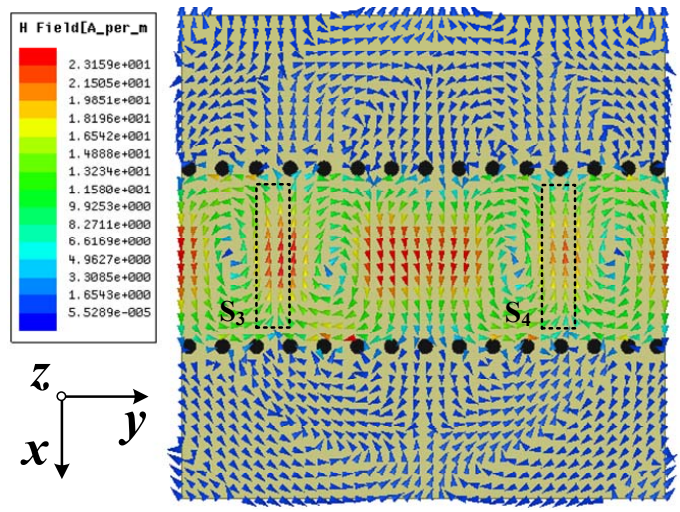

Fig. 15. Magnetic field distribution on top SIW of the slot coupler at 24 $\mathrm{GHz}$.

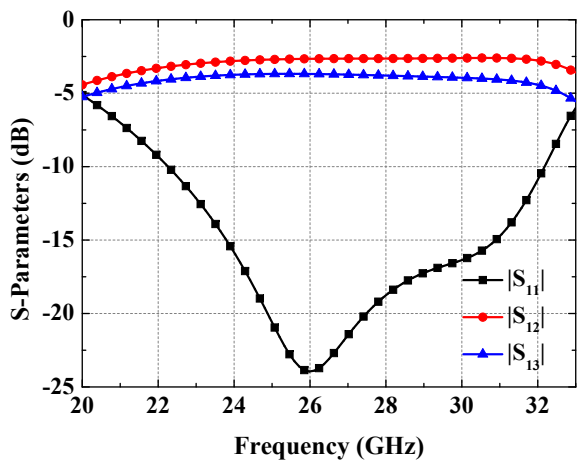

(a)

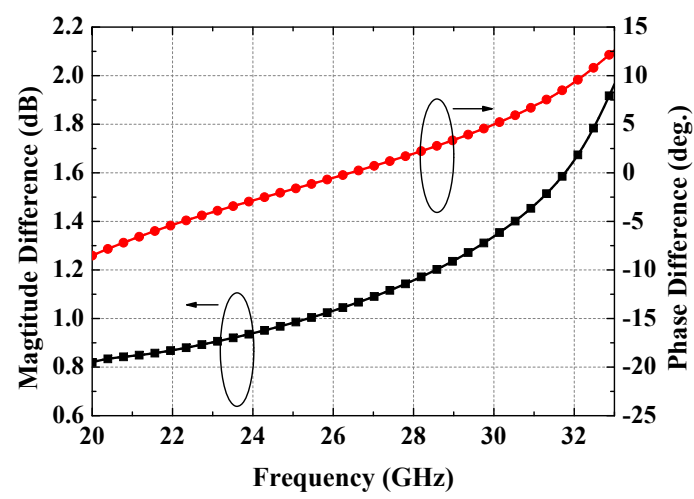

(b)

Fig. 16. Simulated results of the 1-to-2 power divider based on slot coupling. (a) S-parameters. (b) Magnitude and phase imbalances.

The magnetic field distribution in Region II is plotted in Fig. 15. Unlike the previous occasion, the magnetic field mainly has $\mathrm{x}$-component at the coupling aperture and is symmetrical with regard to the coupling slot in y direction. This leads to same magnetic field distributions at the two slots (namely the 


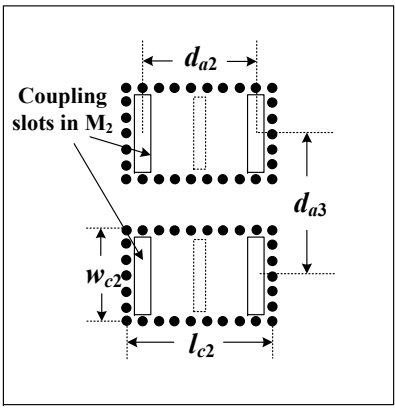

(a)

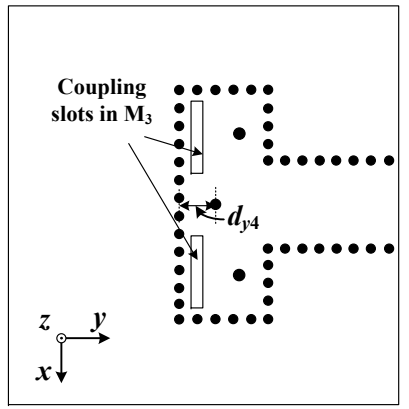

(b)
Fig. 17. Geometrical configuration of the feed network. (a) Top view of Region II. (b) Top view of Region III.

TABLE V

PARAMETERS OF THE ANTENNA ARRAY BASED ON SLOT COUPLING. (UNIT: MM).

\begin{tabular}{cc|cc|cc|cc}
\hline \hline$a_{1}$ & 7.6 & $b_{1}$ & 3.9 & $a_{2}$ & 9.2 & $b_{2}$ & 5.5 \\
\hline \hline$a_{3}$ & 10.8 & $b_{3}$ & 7.1 & $a_{4}$ & 10.8 & $b_{4}$ & 8.7 \\
\hline \hline$D_{1}$ & 0 & $D_{2}$ & 0.6 & $D_{3}$ & 0.9 & $D_{4}$ & 1.2 \\
\hline \hline$d_{a 2}$ & 10.2 & $d_{a 3}$ & 8.8 & $w_{c 2}$ & 7 & $l_{c 2}$ & 11.3 \\
\hline \hline$h$ & 1.016 & $d_{y 4}$ & 2.8 & $w_{s}$ & 1.3 & $l_{s}$ & 6 \\
\hline \hline
\end{tabular}

slots $S_{3}$ and $S_{4}$ labelled in Fig. 15). The same magnetic field distributions indicate that the receiving signals at Port 2 and Port 3 are in-phase. Simulated results of the power divider are plotted in Fig. 16. It is observed from Fig. 16 (a) that the power divider also achieves over $30 \%$ impedance bandwidth with $\left|S_{11}\right|$ below $-10 \mathrm{~dB}$ in the frequency range from 22.3 to $32.2 \mathrm{GHz}$. Fig. 16 (b) shows that the output signals of the power divider are equal in both magnitude and phase across the obtained band.

2) Design of Four Elements Array: Since in-phase power divider is obtained by using slot coupling, the slot radiators can be simply placed symmetrically respect to the coupling slot, as shown in Fig. 15. The resultant feed network of the antenna array consisting both Regions II and III is illustrated in Fig. 17. As shown in Fig. 17 (a), every two rectangular slots form a subarray and are surrounded by a cavity with size of $w_{c 2} \times$ $l_{c 2}$. The adjacent slots are horizontally and vertically spaced by $d_{a 2}$ and $d_{a 3}$, respectively. As illustrated in Fig. 17 (b), a conventional T-shaped 1-to-2 power divider is also designed in Region III to feed the two second-stage 1-to-2 power dividers. A metal post of diameter $1.6 \mathrm{~mm}$ is inserted in the cavity with a distance of $d_{y 4}$ to the sidewall to realize good transmission characteristic of the T-shaped power divider.

3) Experimental Results: Based on the above analyses, a prototype of the obtained array with the dimensions listed in Table $\mathrm{V}$ is fabricated and measured. Fig. 18 shows the photograph of the fabricated prototype. The array is connected to a SMA through a transition from the SIW input in Region III to a 50-Ohms-CPW with a total loss less than $1.9 \mathrm{~dB}$ to facilitate the measurement of antenna performance [20].

The simulated and measured reflection coefficients are com-

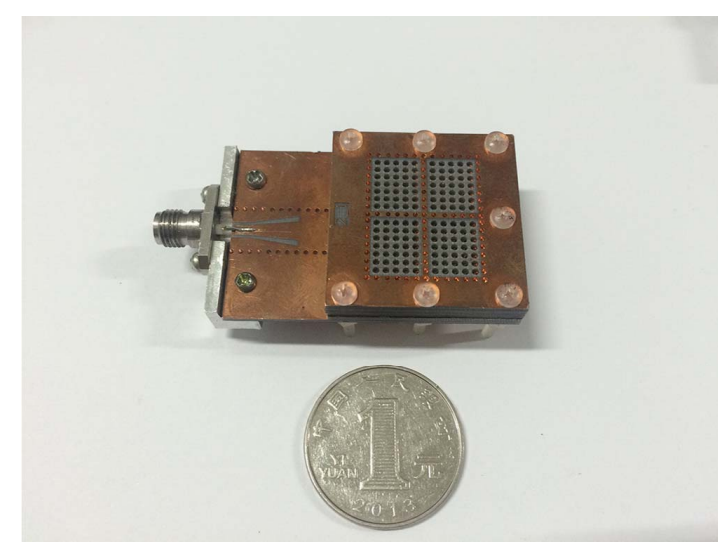

Fig. 18. Photograph of the fabricated antenna array using slot coupling.

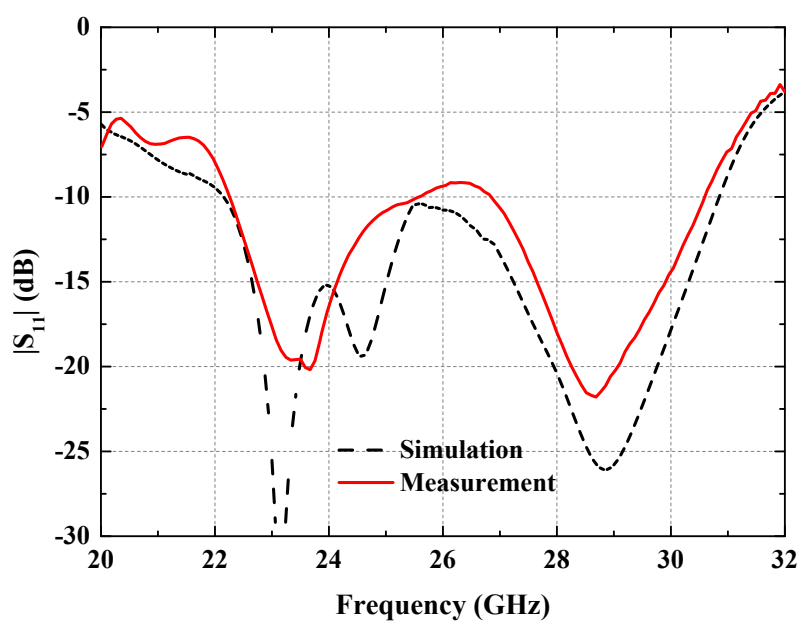

Fig. 19. Simulated and measured reflection coefficients of the proposed antenna array using slot coupling.

pared in Fig. 19. It is observed that the simulated and measured results are generally in good agreement, achieving a wide impedance bandwidth over $30 \%$ ranging from 22.5 to 30.5 GHz. As the air gap still exists in the multilayer structure, the discrepancy between simulated and measured results is generated.

The simulated and measured radiation patterns in both Eand H-planes are shown in Fig. 20. As shown in the figures, the measured results show good accordance with the simulated ones. Above all, the direction of the mainlobe keeps pointing to the broadside direction in the whole operating band due to the symmetrical feed network. Therefore, as depicted in Fig. 21, in comparison with the above result, a more flat gain response with a variation of $1 \mathrm{~dB}$ over the whole operating band is obtained.

Comparisons between some representative works mentioned in the introduction and this work are illustrated in Table VI. As the main concern in this work, the bandwidth is significantly increased compared to other works. Moreover, this work has a very high radiation efficiency due to the loaded multilayer cavities. 
TABLE VI

COMPARISON OF SEVERAL REPRESENTATIVE SIW CAVITY-BACKED SLOT ANTENNA ARRAYS.

\begin{tabular}{|c|c|c|c|c|c|c|}
\hline Ref. & $\begin{array}{c}\text { Center } \\
\text { Frequency }(\mathrm{GHz})\end{array}$ & $\begin{array}{l}\text { Number of } \\
\text { Elements }\end{array}$ & $\begin{array}{c}\text { Impedance } \\
\text { Bandwidth (\%) }\end{array}$ & $\begin{array}{c}\text { 3-dB Gain } \\
\text { Bandwidth (\%) }\end{array}$ & $\begin{array}{c}\text { Peak Gain } \\
\text { (dBi) }\end{array}$ & $\begin{array}{c}\text { Radiation } \\
\text { Efficiency }(\%)\end{array}$ \\
\hline$[3]$ & 60 & $2 \times 4$ & 11.5 & 11.5 & 12 & N/A \\
\hline$[4]$ & 35 & $2 \times 2$ & 13.4 & 13.4 & 10.8 & N/A \\
\hline [8] & 60 & $8 \times 8$ & 17.1 & 17.1 & 22.1 & $\geq 44.4$ \\
\hline [9] & 20 & $4 \times 4$ & 14 & 6.7 & 17.8 & N/A \\
\hline [10] & 20 & $16 \times 16$ & 19.2 & 15 & 29.1 & $\geq 62$ \\
\hline [11] & 8.93 & $4 \times 4$ & 16.7 & 16.7 & 15.5 & $\geq 84$ \\
\hline This work : probe coupling array & 24 & $2 \times 2$ & 33.3 & 29.7 & 14.1 & $\geq 90$ \\
\hline This work : slot coupling array & 26.5 & $2 \times 2$ & 30.2 & 30.2 & 14.2 & $\geq 90$ \\
\hline
\end{tabular}

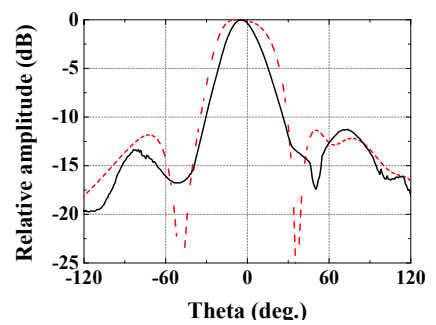

(a)

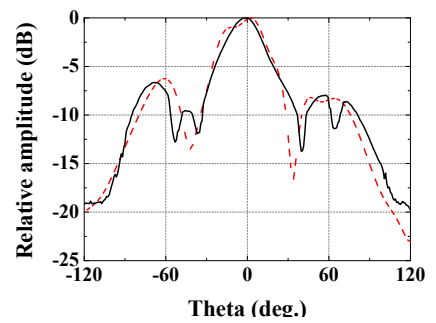

(c)

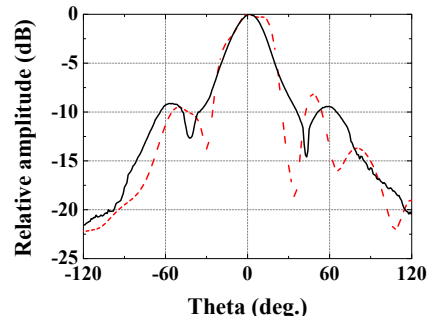

(e)

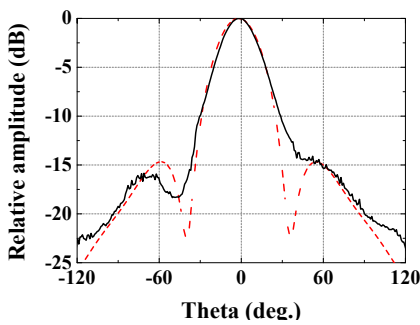

(b)

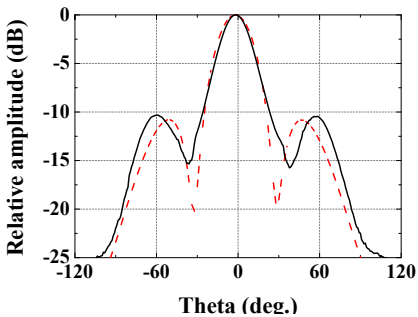

(d)

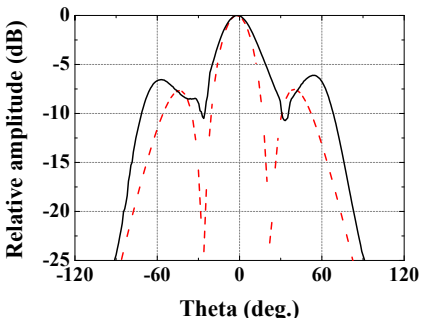

(f)

Fig. 20. Simulated and measured radiation patterns of the fabricated antenna array in two cutting planes at three representing frequencies. (a) $22.5 \mathrm{GHz}$ in E-plane. (b) $22.5 \mathrm{GHz}$ in H-plane. (c) $26.5 \mathrm{GHz}$ in E-plane. (d) $26.5 \mathrm{GHz}$ in H-plane. (e) $30.5 \mathrm{GHz}$ in E-plane. (f) $30.5 \mathrm{GHz}$ in H-plane.(Dashed lines denote the simulated results while solid lines denote measured ones.)

\section{CONCLUSION}

In this papar, we propose a technique to build a wideband SIW $2 \times 2$ elements array. A multilayer SIW cavity-backed slot antenna is adopted as the array element and bandwidth enhancement is further investigated. Based on the wideband element, the feed network using probe coupling and slot coupling is researched, respectively. Results indicate that both the antennas array using different feed network can achieve

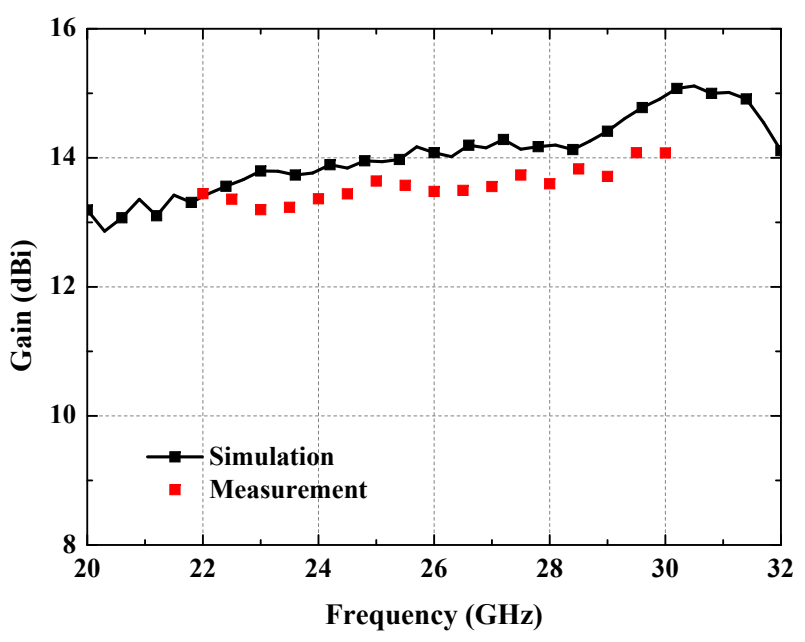

Fig. 21. Simulated and measured broadside gains of the four elements array.

over $30 \%$ impedance bandwidth. In terms of the radiation performance, the proposed antenna array fed by slot-coupling power divider can maintain the mainlobe pointing to the broadside direction, which consequently brings a more flat and higher broadside gain across the whole operating band. Above all, the proposed antenna array features an over $30 \%$ operating bandwidth and can be easily expanded to a largescale antenna array, which will greatly enrich the employment of SIW antenna array in modern communication systems with requirements of sufficient bandwidth and high radiation performance.

\section{ACKNOWLEDGMENT}

The authors would like to thank the reviewers for their inspiring comments and their contribution in correcting the errors in this paper.

\section{REFERENCES}

[1] D. Deslandes, and K. Wu, "Integrated microstrip and rectangular waveguide in planar form," IEEE Microw. and Wireless Compon. Lett., vol. 11, no. 2, pp. 68-70, Feb. 2001.

[2] G.-Q. Luo, "Low profile cavity backed antennas based on substrate integrated waveguide technology," IEEE APCAP, Singapore, Aug. 2012. 
[3] K. Gong, Z.-N. Chen, X.-M. Qing, Z. Song, P. Chen, and W. Hong, "Empirical formula of cavity dominant mode frequency for $60-\mathrm{GHz}$ cavity backed wide slot antenna," IEEE Trans. Antennas Propag., vol. 61, no. 2, pp. 1698-1704, Feb. 2013.

[4] Y. Zhang, Z.-N. Chen, X.-M. Qing, and W. Hong, "Wideband millimeterwave substrate integrated waveguide slotted narrow-wall fed cavity antennas," IEEE Trans. Antennas Propag., vol. 59, no. 5, pp. 1488-1496, May 2011.

[5] K. Wu, Y.-J. Cheng, T. Djerafi, and W. Hong, "Substrate-integrated millimeter-wave and terahertz antenna technology," Proceedings of the IEEE, vol. 100, no. 7, pp. 2219-2232, Jul. 2012.

[6] L. Xu, L. Li, and W.-M. Zhang, "Study and design of broadband bow-tie slot antenna fed with asymmetric CPW," IEEE Trans. Antennas Propag., vol. 63, no. 2, pp. 760-765, Feb. 2015.

[7] A. A. Eldek, A. Z. Elsherbeni, and C. E. Smith, "Wideband microstrip-fed printed bow-tie antenna for phased-array systems," Microw. Opt. Technol. Lett., vol. 43, no. 2, pp. 123-126, Oct. 2004.

[8] K. Kiminami, A. Hirata, and T. Shiozawa, "Double sided printed bowtie antenna for UWB communications," IEEE Antennas Wireless Propag. Lett., vol. 3, pp. 152-153, 2004.

[9] M. V. Varnoosfaderani, D. V. Thiel, and J.-W Lu, "A wideband slot antenna in a box for wearable sensor nodes," IEEE Antennas Wireless Propag. Lett., vol. 14, pp. 1494-1497, 2015.

[10] S.-W. Qu, J.-L. Li, Q. Xue, and C.-H, Chan, "Wideband periodic endfire antenna with bowtie dipoles," IEEE Antennas Wireless Propag. Lett., vol. 7, pp. 314-317, 2008 .

[11] A. Dastranj, A. Imani, and M. Naser-Moghaddasi, "Printed wide-slot antenna for wideband applications," IEEE Trans. Antennas Propag., vol. 56, no. 10, pp. 3097-3102, Oct. 2008.

[12] S. Yun, D. Kim, and S. Nam, "Bandwidth enhancement of cavity-backed slot antenna using a via-hole above the slot," IEEE Antennas Wireless Propag. Lett., vol. 11, pp. 1092-1095, 2012.

[13] G.-Q. Luo, Z.-F. Hu, W.-J. Li, X.-H. Zhang, L.-L. Sun, and J.-F. Zheng, "Bandwidth-enhanced low-profile cavity-Backed slot antenna by using hybrid SIW cavity modes," IEEE Trans. Antennas Propag., vol. 60, no. 4, pp. 1698-1704, Jan. 2012.

[14] J.-F. Xu, Z.-N. Chen, X.-M. Qing, and W. Hong, "Bandwidth enhancement for a $60 \mathrm{GHz}$ substrate integrated waveguide fed cavity array antenna on LTCC," IEEE Trans. Antennas Propag., vol. 59, no. 3, pp. 826-832, Mar. 2011.

[15] D.-F. Guan, Z.-P. Qian, Y.-S. Zhang, and Y. Cai, "Novel SIW cavity backed antenna array without using individual feeding network," IEEE Antennas Wireless Propag. Lett., vol. 13, pp. 423-426, 2014.

[16] D.-F. Guan, C. Ding, Z.-P. Qian, Y.-S. Zhang, W.-Q. Cao, and E. Dutkiewicz, "An SIW based large-scale corporate-feed array antenna," IEEE Trans. Antennas Propag., vol. 63, no. 7, pp. 2969-2976, Jul. 2015.

[17] P. Wu, S. Liao, and Q. Xue, "A substrate integrated slot antenna array using simplified feeding network based on higher order cavity modes," IEEE Trans. Antennas Propag., vol. 64, no. 1, pp. 126-135, Jan. 2016.

[18] Y. Cai, Z.-P. Qian, Y.-S. Zhang, J. Jin, and W.-Q. Cao, "Bandwidth enhancement of SIW horn antenna loaded with air-via perforated dielectric slab," IEEE Antennas Wireless Propag. Lett., vol. 13, pp. 571-574, 2014.

[19] Y. Cai, Y.-S. Zhang, Z.-P. Qian, W.-Q. Cao, and L. Wang, "Design of compact air-vias-perforated SIW horn antenna with partially detached broad walls," IEEE Trans. Antennas Propag., vol. 64, no. 6, pp. 21002107, Jun. 2016.

[20] R. Kazemi, A. E. Fathy, S. N. Yang, and R. A. Sadeghzadeh, "Development of an ultra wide band GCPW to SIW transition," in Proc. Radio Wireless Symp. (RWS), Jun. 2012, pp. 171-174.

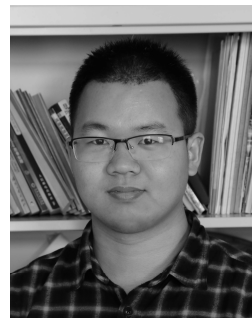

Yang Cai was born in Anhui, China, in 1991. He received the B.S. degree in communication engineering from the PLA University of Science and Technology, Nanjing, China, in 2012.

His research interests include substrate integrated waveguide antennas, horn antennas, metamaterialsbased antennas and array applications.

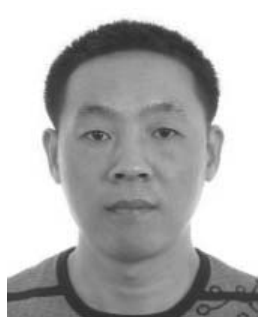

Yingsong Zhang was born in Hebei, China, in 1973. $\mathrm{He}$ received the B.S. and M.S. degrees in electromagnetic field and microwave technology from the PLA Institute of Communications Engineering, Nanjing, China, in 1996 and 1999, respectively, and the Ph.D. degree in electromagnetic field and microwave technology from the Southeast University, Nanjing, China, in 2013

He has authored or coauthored more than 30 international and regional refereed journal papers. His research interests include multibeam lens antenna, large-scale array antenna, and passive components based on novel transmission lines.

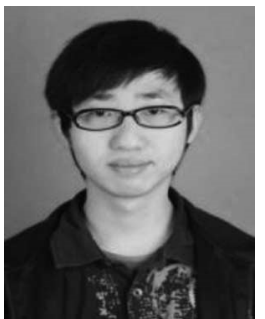

Can Ding was born in Anhui, China, in 1989. He received the B.S. degree in micro-electronics from Xidian University, Xian, China, in 2009. From 2010 to 2011, he had been working toward the Masters degree in micro-electronics from Xidian University and then transferred to Ph.D. He is currently under the cotutelle agreement between Macquarie University, Sydney, N.S.W., Australia and Xidian University. $\mathrm{He}$ is also with CSIRO DPaS Flagship, Marsfield, N.S.W., Australia. He is now working as a Lecturer in UTS.

His research interests include reconfigurable antenna, phased array antenna, phase shifter, and microwave circuit design.

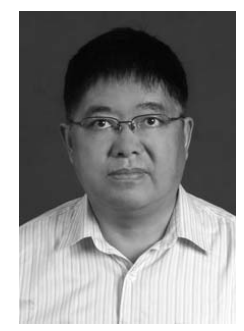

Zuping Qian was born in Jiangsu, China, in 1961. $\mathrm{He}$ received the B.S. and M.S. degrees in applied mathematics from the Hunan University, Changsha, China, in 1982 and 1985, respectively, and the Ph.D. degree in microwave techniques from the Southeast University, Nanjing, China, in 2000.

During 1985C1999, he worked with the Institute of Communications Engineering (ICE), Nanjing, China, as a Lecturer and later as an Associate Professor. Since 2000, he has been a Professor with the College of Communications Engineering, PLA University of Science and Technology, Nanjing, China. He has authored or coauthored more than 80 international and regional refereed journal papers. His research interests include antenna, metamaterials, computational electromagnetics, array signal processing, and EMI/EMC. 